\title{
A bounded confidence model that preserves the signs of the opinions
}

Francesca Ceragioli, Gustav Lindmark, Veibäck Clas, Niklas Wahlström, Martin Lindfors and Claudio Altafini

Book Chapter

Tweet

N.B.: When citing this work, cite the original article.

Part of: Proceedings of the 2016 European Control Conference (ECC), 2016, pp. 543-548. ISBN: 9781509025916 (online), 9781509025909 (USB), 9781509025923 (PoD)

DOI: http://dx.doi.org/10.1109/ECC.2016.7810341

Copyright: Institute of Electrical and Electronics Engineers (IEEE)

Available at: Linköping University Electronic Press

http://urn.kb.se/resolve?urn=urn:nbn:se:liu:diva-133962

LINKÖPINGS

UNIVERSITET 


\title{
A bounded confidence model that preserves the signs of the opinions
}

\author{
Francesca Ceragioli, Gustav Lindmark, Clas Veibäck, Niklas Wahlström, Martin Lindfors, and Claudio Altafini
}

\begin{abstract}
The aim of this paper is to suggest a modification to the usual bounded confidence model of opinion dynamics, so that "changes of opinion" (intended as changes of the sign of the initial state) of an agent are never induced by the dynamics. In order to do so, a bipartite consensus model is used, endowing it with a confidence range. The resulting signed bounded confidence model has a state-dependent connectivity and a behavior similar to its standard counterpart, but in addition it preserves the sign of the opinions by "repelling away" opinions localized near the origin but on different sides with respect to 0 .
\end{abstract}

\section{INTRODUCTION}

A bounded confidence model is a model of consensuslike opinion dynamics in which the agents interact with each other only when their opinions are close enough. Such a class of models usually go under the name of HegselmannKrause models [12] and have the peculiarity of expressing confidence as a function of the distance between the agents' states. As a consequence, the graph that describes the interactions between the agents is itself state-dependent and varying in time. The emerging behaviour of such a model is that the agents tend to form clusters, and a consensus value is achieved within the agents participating to a cluster. In the control literature, various aspects of such models have been studied: discrete-time [4], continuous-time [5], and stochastic [7] behaviour, existence of interaction rules that allow to preserve the connectivity [13], presence of stubborn agents [10] etc. See [9], [11] for an overview. In continuous time, if the confidence range is delimited by a sharp threshold, then the right hand side of the resulting ODEs is discontinuous. Existence and uniqueness analysis of the corresponding solutions have been carried out in [5], [6]. In [6] approximations of the discontinuous dynamics are suggested.

The basic reason to bound the confidence range is that agents with similar opinions are more likely to trust each other. Mediation to a common consensus value then does not require widely different opinions to converge to a common value, and allows for "persistent disagreement" among different groups to be maintained asymptotically. While the notion of bounded consensus is plausible and acceptable in most contexts, there is a specific situation in which confidence between the agents may be lost even if the opinions are

F. Ceragioli is with Dip. di Mathematica, Politecnico di Torino, Corso Duca degli Abruzzi, 24, 10129 Italy.

G. Lindmark, C. Veibäck, N. Wahlström, M. Lindfors and C. Altafini are with the Division of Automatic Control, Dept. of Electrical Engineering, Linköping University, SE-58183, Linköping, Sweden. email: claudio.altafini@liu.se in proximity, and it is when their signs are different. It is intuitively clear that "changing opinion", intended as changing sign of an agent's opinion, is a fairly drastic process, a "mental barrier" not so likely to be trespassed in real scenarios.

When computing consensus among the agents participating in a cluster, currently available bounded confidence models do not distinguish between the case of all opinions having the same sign or less, i.e., the opinions can freely cross zero while converging to a local consensus value.

The aim of this paper is to propose a model of bounded confidence which preserves the signs of the original opinions. To do so, we make use of the notion of bipartite consensus introduced in [1]. Its main feature is that in presence of a graphical property called structural balance, the nodes of a graph can be split into two subgroups, with the nodes within each subgroup being connected only through positive edges ("friends") while the edges between nodes on different subgroups are all negative ("enemies"). Using the corresponding signed Laplacian to perform a consensus-like protocol leads to the nodes converging all to a common value in modulus but not in sign, with each group of the partition polarizing to a different sign.

When applying bipartite consensus to a confidence model, it is quite obvious what the partition of the nodes (and hence the signs of the edges of the graph of interactions) should be: initial opinions that have the same sign correspond to positive edges, while those having opposite signs correspond to negative edges. In this way the graph of interactions is always structurally balanced by construction. The bipartite consensus rule implies then that opinions of opposite sign "repel" each other. This is imposed in the model through a sign function, meaning that even when no bound on the confidence is present, the (unbounded) signed confidence model has state-dependent connectivity. In spite of a discontinuous right-hand side, this model almost always has unique solutions. Only when one or more of the initial opinions are 0, then multiple Carathéodory solutions arise.

When a bound is added on the confidence range, then the repulsions among agents are only localized around the origin and do not affect the asymptotic behavior of opinions far from 0 . Even with the extra repulsion around the origin, the overall behavior of the model is to create clusters of agents, and to achieve a common consensus value within a cluster for almost all initial solutions. What our model guarantees in addition is that the sign of the initial conditions is respected, i.e., no agent has to change his/her mind during the time evolution of the system. 


\section{BACKGROUND MATERIAL}

\section{A. Linear algebraic notions}

A matrix $A \in \mathbb{R}^{n \times n}$ is said Hurwitz stable if all its eigenvalues $\lambda_{i}(A), i=1, \ldots, n$, have $\operatorname{Re}\left[\lambda_{i}(A)\right]<0$. It is said marginally stable if $\operatorname{Re}\left[\lambda_{i}(A)\right] \leqslant 0, i=1, \ldots, n$, and $\lambda_{i}(A)$ such that $\operatorname{Re}\left[\lambda_{i}(A)\right]=0$ have an associated Jordan block of order one. $A$ is said irreducible if there does not exist a permutation matrix $\Pi$ such that $\Pi^{T} A \Pi$ is block triangular. The matrices $A$ considered in this paper will always be symmetric: $A=A^{T}$.

\section{B. Bipartite Consensus}

Given a matrix $A$ of elements $A_{i j}$, the (standard) Laplacian associated with $A$ is the matrix $L$ of elements

$$
L_{i j}= \begin{cases}-A_{i j} & \text { if } i \neq j \\ \sum_{k \neq i} A_{i k} & \text { if } i=j .\end{cases}
$$

The signed Laplacian $L_{s}$ is given by

$$
L_{s, i j}= \begin{cases}-A_{i j} & \text { if } i \neq j \\ \sum_{k \neq i}\left|A_{i k}\right| & \text { if } i=j .\end{cases}
$$

When $A \geq 0$ the two definitions coincide. In any case, the two Laplacians are diagonally equipotent matrices. $L$ is always singular, while $L_{s}$ may or may not be [1], [2].

Given a matrix $A=A^{T} \in \mathbb{R}^{n \times n}$, consider the undirected graph $\Gamma(A)$ of $A: \Gamma(A)=\{\mathcal{V}, A\}$ where $\mathcal{V}=\{1, \ldots, n\}$ is the set of $n$ nodes and $A$ is its weighted adjacency matrix. $\Gamma(A)$ is connected if $\exists$ a path between each pair of nodes in $\mathcal{V}$. When $A$ can assume both positive and negative values, then $\Gamma(A)$ is said a signed graph. An undirected signed graph $\Gamma(A)$ is said structurally balanced if all its cycles are positive (i.e., they have an even number of negative edges). $\Gamma(A)$ is structurally balanced if and only if $L_{s}$ is a singular matrix, see [1], [2]. In this case, $\exists$ vector $s=\left[\begin{array}{lll}s_{1} & \cdots & s_{n}\end{array}\right], s_{i}=$ \pm 1 , such that $L=S L_{s} S$, where $S=\operatorname{diag}(s)$ is the diagonal matrix having the entries of $s$ on the diagonal. If $A \geq 0$ and irreducible, a linear consensus problem is given by the linear system

$$
\dot{x}=-L x \text {. }
$$

Since $L$ diagonally equipotent and singular, its solution is

$$
x^{*}=\frac{\sum_{j} x_{j}(0)}{n} \mathbb{1}
$$

where $\mathbb{1}$ is the right eigenvector relative to $\lambda_{1}(L)=0$. If $A$ can have negative entries and $\Gamma(A)$ is structurally balanced, then a bipartite consensus problem is given by the following linear system:

$$
\dot{x}=-L_{s} x
$$

whose solution is

$$
x^{*}=\frac{\sum_{j}\left|x_{j}(0)\right|}{n} S \mathbb{1}
$$

corresponding to a bipartite consensus value: $\left|x_{i}^{*}\right|=\left|x_{j}^{*}\right|$.

\section{Solutions of ODEs}

Given $g: \mathbb{R}^{n} \rightarrow \mathbb{R}^{n}$, the system

$$
\dot{x}=g(x), \quad x(0)=x_{o}
$$

a classical solution of (4) on the interval $\left[0, t_{1}\right)$ is a map $\phi:\left[0, t_{1}\right) \rightarrow \mathbb{R}^{n}$ such that

1) $\phi$ is differentiable in $\left[0, t_{1}\right)$,

2) $\phi(0)=x_{o}$

3) $\dot{\phi}(t)=g(\phi(t))$ for all $t \in\left[0, t_{1}\right)$.

When a function satisfies the equation (4) except perhaps for a set of measure zero, then we can use the notion of Carathéodory solution. More formally, a Carathéodory solution of (4) on the interval $\left[0, t_{1}\right)$ is a map $\phi:\left[0, t_{1}\right) \rightarrow$ $\mathbb{R}^{n}$ such that

1) $\phi$ is absolutely continuous in $\left[0, t_{1}\right)$,

2) $\phi(0)=x_{o}$

3) $\dot{\phi}(t)=g(\phi(t))$ for almost all $t \in\left[0, t_{1}\right)$.

Equivalently, a Carathéodory solution of (4) is a solution of the integral equation

$$
x(t)=x_{o}+\int_{0}^{t} g(x(\tau)) d \tau .
$$

See [8], [6] for more details.

\section{Bounded Confidence}

A bounded confidence model is given by the following consensus-like scheme in $\mathbb{R}^{n}$

$$
\dot{x}_{i}(t)=\sum_{j \text { s.t. }\left|x_{j}(t)-x_{i}(t)\right|<1}\left(x_{j}(t)-x_{i}(t)\right)
$$

The interpretation of (5) is that only nodes whose opinion is closed enough to that of node $i$ contribute to the summation at each $t$.

If $\Gamma(A(x(t)))=\{\mathcal{V}, A(x(t))\}$ is the graph given by the pattern of active connections of (5) at time $t \geq 0$, then for the adjacency matrix $A(x(t))$ one has $A_{i j}(x(t))=1 \Longleftrightarrow$ $\left|x_{j}(t)-x_{i}(t)\right|<1 . A(x(t))$ is in general time-varying and discontinuous in time.

The behavior of (5) is well-known. For example, we have that in spite of the discontinuous right hand side, (5) has a unique Carathéodory solution for almost all initial conditions. Following [5], we say that $x_{o} \in \mathbb{R}^{n}$ is a proper initial condition if it satisfies the following conditions:

1) $\exists$ a unique $x: \mathbb{R}^{+} \rightarrow \mathbb{R}^{n}, t \rightarrow x(t)$ satisfying

$$
x_{i}(t)=x_{i}(0)+\int_{0}^{t} \sum_{\substack{j \text { s.t. } \\\left|x_{i}-x_{j}\right|<1}}\left(x_{j}(\tau)-x_{i}(\tau)\right) d \tau, \quad x(0)=x_{o} ;
$$

2) the subset of $\mathbb{R}^{+}$on which $x$ is not differentiable is at most countable and has no accumulation point;

3) $x_{i}(t)=x_{j}(t) \Longrightarrow x_{i}\left(t^{\prime}\right)=x_{j}\left(t^{\prime}\right) \forall t^{\prime} \geq t$;

In the terminology of [5], proper initial conditions yield proper Carathéodory solutions of (6). It is shown in [5] that except for at most a set of Lebesgue measure zero all initial conditions are proper initial conditions. This is listed 
as property $\mathrm{P} 1$ in the following Theorem, that summarizes the behavior of the bounded confidence model of [5].

Theorem 1 Consider the system (5). Its solutions have the following properties:

P1: Almost all $x_{o} \in \mathbb{R}^{n}$ are proper initial conditions.

Furthermore, for solutions issuing from proper initial conditions:

P2: $x_{i}(\tau) \leq x_{j}(\tau) \Longrightarrow x_{i}(t) \leq x_{j}(t) \forall t \geq \tau ;$

P3: The average opinion

$$
c=\frac{1}{n} \sum_{i} x_{i}(t)
$$

is constant $\forall t \geq 0$;

P4: The sum of squared differences from the average $W(x(t))=\sum_{i}\left(x_{i}(t)-c\right)^{2}$ is non-increasing and $\frac{d W}{d t}$ is almost always negative;

P5: If $\exists \tau \geq 0$ such that $\Gamma(A(\tau))$ is fully connected, then $\Gamma(A(t))$ fully connected $\forall t \geq \tau$.

P6: If $\exists \tau \geq 0$ such that $\Gamma(\bar{A}(\tau))$ is not connected, then $\Gamma(A(t))$ not connected $\forall t \geq \tau$.

P7: $\lim _{t \rightarrow \infty} x(t)=x^{*}$ where $x^{*}$ is such that $\forall i, j \in$ $\{1, \ldots, n\}$ either $x_{i}^{*}=x_{j}^{*}$ or $\left|x_{i}^{*}-x_{j}^{*}\right| \geq 1$.

For all properties, a proof is available in the literature (see e.g. [5], [6], [13]) or immediately deducible from it. Hence it is omitted here. The meaning of properties P4-P7 is that opinions tend to cluster into "local" consensus values distant at least 1 from each other. In a model like (5), the sign of the opinions does not matter, i.e., nearby opinions of different sign are treated as those of equal sign.

\section{Signed CONFIDENCE AS BIPARTITE CONSENSUS}

In this section we present a model of (unbounded) confidence in which changes of sign of the opinions are avoided. We call this problem signed confidence. The model we consider for this is the following:

$$
\dot{x}_{i}(t)=\sum_{j \neq i}\left(\operatorname{sgn}\left(x_{j}(t) x_{i}(t)\right) x_{j}(t)-x_{i}(t)\right)
$$

where $\operatorname{sgn}(\cdot)$ is the sign function

$$
\operatorname{sgn}(z)= \begin{cases}1 & \text { if } z>0 \\ 0 & \text { if } z=0 \\ -1 & \text { if } z<0\end{cases}
$$

The presence of the sign function means that the system (8) has a discontinuous right hand side when one or more of the $x_{i}$ are equal to 0 . It will sometimes be convenient to write the equations (8) as

$$
\dot{x}=f(x)
$$

where $f: \mathbb{R}^{n} \rightarrow \mathbb{R}^{n}$ is the discontinuous vector field of components $f_{i}(x)=\sum_{j \neq i}\left(\operatorname{sgn}\left(x_{j} x_{i}\right) x_{j}-x_{i}\right)$. The following proposition shows that in spite of the discontinuities, the solutions of (9) corresponding to almost all initial conditions exist and can be continued to the whole half line, i.e., almost all initial conditions are proper.

\section{Proposition 1 The system (8) satisfies property $P 1$.}

Proof: In order to prove that solutions corresponding to almost all initial conditions exist and that they can be continued on a whole half line, we prove that the discontinuity surfaces $x_{i}=0$ are repellent with respect to at least one of the limit values of the vector field $f(\cdot)$. Consider the function $\sigma_{i}(x)=x_{i}$ and the surface $\sigma_{i}(x)=0$. Let $x$ be a point of this surface and let $f^{-}(x)$ the limit value of $f(x)$ as $x$ approaches the surface with $x_{i}<0$. It holds

$$
\nabla \sigma_{i}(x) \cdot f^{-}(x)=-\sum_{j \neq i}\left|x_{j}\right|<0
$$

if at least one $j$ is such that $x_{j} \neq 0$, i.e. the point does not coincide with the origin. Analogously if $f^{+}(x)$ is the limit value of $f(x)$ as $x$ approches the surface with $x_{i}>0$ one has

$$
\nabla \sigma_{i}(x) \cdot f^{+}(x)=\sum_{j \neq i}\left|x_{j}\right|>0
$$

if at least one $j$ is such that $x_{j} \neq 0$. This means that solutions issuing from points with $x_{i} \neq 0$ for all $i$ cannot reach the discontinuity surfaces.

A consequence of Proposition 1 is the following.

Proposition 2 Consider the system (8). If $\forall i=1, \ldots, n$ $x_{i}(0) \neq 0$ then $\operatorname{sgn}\left(x_{i}(t)\right)=\operatorname{sgn}\left(x_{i}(0)\right) \forall t \in[0, \infty)$ and $\forall i=1, \ldots, n$.

Proof: Consider first the case $x_{i}(0) \neq 0 \forall i=1, \ldots, n$. Assuming without loss of generality that for the $x_{i}(t)$ sorted in absolute value it holds $0<\left|x_{1}(t)\right| \leq\left|x_{2}(t)\right| \leq \ldots \leq$ $\left|x_{n}(t)\right|$, if $x_{1}(t)>0$, then

$$
\begin{aligned}
\dot{x}_{1} & =\sum_{j}\left(\operatorname{sgn}\left(x_{j}(t) x_{1}(t)\right) x_{j}(t)-x_{1}(t)\right) \\
& =\sum_{j}\left(\left|x_{j}(t)\right|-\left|x_{1}(t)\right|\right) \geq 0,
\end{aligned}
$$

and analogously for $x_{1}(t)<0$. In both cases, $x_{1}(t)$ is repelled from the origin, (or at least does not approach it), meaning that $\operatorname{sgn}\left(x_{i}(0)\right)=\operatorname{sgn}\left(x_{i}(t)\right) \forall t \geq 0$ and $\forall i=1, \ldots, n$.

Remark 1 From (10), if $x_{i}(0) \neq 0 \forall i=1, \ldots, n$, it is straightforward to show that the following conservation law holds for the system (8):

$$
c_{s}=\frac{1}{n} \sum_{j}\left|x_{j}(0)\right|=\frac{1}{n} \sum_{j}\left|x_{j}(t)\right| \quad \forall t \geq 0 .
$$

Note that we can ignore the fact that the absolute value is a nondifferentiable function as $x_{i}(t)$ does not change sign.

On the contrary, $c$ of (7) is not a conservation law, as can be computed directly (omitted for lack of space). As a consequence, it follows that $W(x(t))$ need not be nonincreasing, see Example 1. 
Example 1 Consider the system (8) with the $n=3$ initial opinions $x(0)=\left[\begin{array}{lll}-0.15 & 0.2 & -0.1\end{array}\right]^{T}$. The corresponding $W(x(t))$ is shown in red in Fig. 1. Also $W_{s}(x(t))=$ $\sum_{i}\left(x_{i}(t)-c_{s}\right)^{2}$ is increasing in this example (blue dashed curve in Fig. 1).

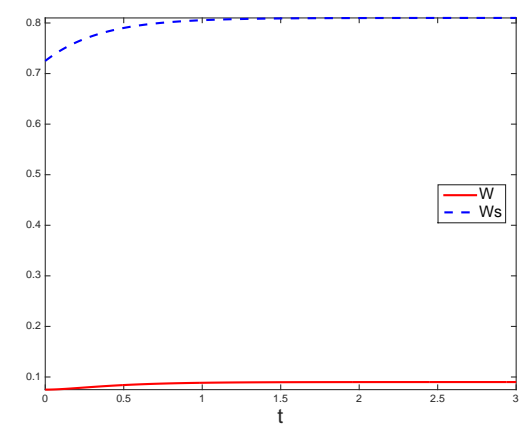

Fig. 1. Example 1: the functions $W(x(t))$ (red, solid) and $W_{s}(x(t))$ (blue, dashed) are both increasing.

Remark 2 When $x_{i}(0) \neq 0 \forall i=1, \ldots, n$, the system (8) corresponds to a fully connected bipartite consensus problem, with bipartition given by $s(x(t))=\operatorname{sgn}(x(t))$. To see it, it is enough to observe that the signed adjacency matrix $A_{s}(x(t))$ of (8) is $A_{s}(x(t))=S(x(t)) A S(x(t))$, $S(x(t))=\operatorname{diag}(s(x(t)))$, where the entries of $A$ are

$$
A_{i j}= \begin{cases}1 & \text { if } i \neq j \\ 0 & \text { if } i=j\end{cases}
$$

and those of $A_{s}(x(t))$ are

$$
A_{s, i j}(x(t))= \begin{cases}\operatorname{sgn}\left(x_{i}(t) x_{j}(t)\right) & \text { if } i \neq j \\ 0 & \text { if } i=j .\end{cases}
$$

From Proposition 2, $s(x(t))=s\left(x_{o}\right) \forall t \geq 0 \Longrightarrow$ $A_{s}(x(t))=A_{s}\left(x_{o}\right) \forall t \geq 0$. Hence $\Gamma_{s}=\left\{\mathcal{V}, A_{s}\right\}$ is a structurally balanced (and constant) graph $\forall t$. If $L$ is the Laplacian of $A$, then the signed Laplacian of $A_{s}(x)$, $L_{s}(x)=S(x) L S(x)$, has entries

$$
L_{s, i j}(x)= \begin{cases}-\operatorname{sgn}\left(x_{i} x_{j}\right) & \text { if } i \neq j \\ n-1 & \text { if } i=j .\end{cases}
$$

It is straightforward to check that (13) and (1) coincide.

Proposition 3 Consider the system (8). If $x_{i}(0) \neq 0 \forall i=$ $1, \ldots, n$, then the system converges to bipartite consensus:

$$
\lim _{t \rightarrow \infty} x_{i}(t)=\operatorname{sgn}\left(x_{i}(0)\right) \frac{\sum_{j}\left|x_{j}(0)\right|}{n} .
$$

Proof: The proof follows from the same change of basis of Remark 2.

From Propositions 2-3 and Remark 2, the case of all nonzero initial conditions behaves exactly like a bipartite linear consensus problem on a structurally balanced graph. The following special case has however no counterpart in linear bipartite consensus. It deals with non-proper initial conditions, in correspondence of which multiple Carathéodory solutions exist.

Proposition 4 Consider the system (8). If $x_{i}(0)=0$ for some $i=1, \ldots, n$, then for $t>0$ the system has multiple Carathéodory solutions, corresponding to the limit values of the $i$-th component of the vector field $f$ as $x_{i} \rightarrow 0$, namely

$$
\dot{x}_{i}=\left\{\begin{array}{l}
f_{i}^{+}(x)=\sum_{j \neq i}\left|x_{j}\right| \\
f_{i}^{0}(x)=0 \\
f_{i}^{-}(x)=-\sum_{j \neq i}\left|x_{j}\right| .
\end{array}\right.
$$

In particular, the solution corresponding to (14b) is a classical solution and corresponds to all opinions collapsing to the origin

$$
\lim _{t \rightarrow \infty} x_{i}(t)=0 \quad \forall i=1, \ldots, n .
$$

The proof is omitted for lack of space (it is based on a Lyapunov function argument).

Example 2 Consider (8) with $n=2$ and initial condition $x_{o}=\left[\begin{array}{ll}0 & 1\end{array}\right]^{T}$. The (classical) solution issuing from $x_{o}$ and corresponding to $f^{0}(\cdot)$ asymptotically tends to $\left[\begin{array}{ll}0 & 0\end{array}\right]^{T}$. Of the two other solutions issuing from $x_{o}$, one tends to the point $[-1 / 21 / 2]^{T}$ and the other to the point $[1 / 21 / 2]^{T}$.

\section{Signed BOUNDED CONFIDENCE}

Combining the model of Section III with bounded confidence gives rise to the following signed bounded confidence model:

$$
\dot{x}_{i}(t)=\sum_{j \text { s.t. }\left|x_{j}(t)-x_{i}(t)\right|<1}\left(\operatorname{sgn}\left(x_{j}(t) x_{i}(t)\right) x_{j}(t)-x_{i}(t)\right)
$$

For later use rewrite (15) as

$$
\dot{x}=h(x)
$$

of components

$$
h_{i}(x)=\sum_{j \text { s.t. }\left|x_{j}(t)-x_{i}(t)\right|<1}\left(\operatorname{sgn}\left(x_{j}(t) x_{i}(t)\right) x_{j}(t)-x_{i}(t)\right) .
$$

Theorem 2 The model (15) satisfies the property P1. For solutions issuing from proper initial conditions, the model (15) satisfies P2 and P7, but not P3, P4, P5, P6. Furthermore, it holds:

P8: If $x_{i}(0) \neq 0 \forall i=1, \ldots, n$, then the average of the absolute values, $c_{s}$, is constant $\forall t \geq 0$.

P9: If $x_{i}(0)=0$ for some $i=1, \ldots, n$, then $\exists$ a solution of (15) such that $x_{i}^{*}=0$.

Proof: To avoid trivial cases, let us assume that $\mathcal{I}_{+} \neq \emptyset$, and $\mathcal{I}_{-} \neq \emptyset$. Let us now show that the model (15) obeys the properties listed in the Theorem.

P1. First of all, let us observe that for the model (15) the opinions are sign invariant for solutions issuing from proper initial conditions. In fact, from Propositions 1 and 4 the discontinuity surfaces $\sigma_{i}(x)=0$ do not correspond to proper 
initial conditions. These surfaces are (locally) repelling even when the confidence is bounded. Furthermore, we can remark that for any initial condition outside $\sigma_{i}(x)=0$ for all $i$, there exists a unique local solution. For such initial conditions Proposition 2 still holds once the summations are reduced to the opinions fulfilling the condition $\left|x_{j}-x_{i}\right|<1$, hence an $x_{i} \neq 0$ can never cross the origin.

We then have to prove that existence and uniqueness of any such solutions is not lost in case it reaches the discontinuity surface at a point $\bar{x}$ such that $\bar{x}_{i}-\bar{x}_{j}=1$ (the case $\bar{x}_{i}-\bar{x}_{j}=$ -1 is analogous). The case $\bar{x}_{i}, \bar{x}_{j}>0$ is the same treated in [3]. We then have to examine the cases $\bar{x}_{i}=1, \bar{x}_{j}=0$ and $\bar{x}_{j}<0<\bar{x}_{i}$.

Considering the first one, as the surfaces $\sigma_{j}(x)=0$ are repelling (Proposition 1), the set of points reaching them has measure zero.

We then consider the case $\bar{x}_{j}<0<\bar{x}_{i}$. Let $\sigma_{i j}(x)=$ $x_{i}-x_{j}, \Sigma_{i j}=\left\{x \in \mathbb{R}^{n}: x_{i}-x_{j}=1\right\}, \Sigma_{i j}^{-}=\left\{x \in \mathbb{R}^{n}:\right.$ $\left.x_{i}-x_{j}<1\right\}$ and $\Sigma_{i j}^{+}=\left\{x \in \mathbb{R}^{n}: x_{i}-x_{j} \geq 1\right\}$. Assume that the solution is approaching the surface $\Sigma_{i j}$ from $\Sigma_{i j}^{-}$. In this case, at $x \in \Sigma_{i j}^{-}$it must be

$$
\begin{aligned}
\nabla \sigma_{i j}(x) \cdot h(x) & =h_{i}(x)-h_{j}(x) \\
& =\sum_{\left|x_{\ell}-x_{i}\right|<1}\left(\left|x_{\ell}\right|-x_{i}\right)-\sum_{\left|x_{k}-x_{j}\right|<1}\left(-\left|x_{k}\right|-x_{j}\right)>0 .
\end{aligned}
$$

Since $x \in \Sigma_{i j}^{-}$, the edge $(i, j)$ is present in $\Gamma_{s}(x)$. Emphasizing it in the previous expression:

$$
\begin{aligned}
\nabla \sigma_{i j}(x) \cdot h(x)= & \sum_{\substack{\left|x_{\ell}-x_{i}\right|<1 \\
\ell \neq j}}\left(\left|x_{\ell}\right|-x_{i}\right)+\left|x_{j}\right|-x_{i} \\
& +\sum_{\substack{\left|x_{k}-x_{j}\right|<1 \\
k \neq i}}\left(-\left|x_{k}\right|-x_{j}\right)-\left(-\left|x_{i}\right|-x_{j}\right)>0 .
\end{aligned}
$$

Consider the situation of at least one of the nodes $i$ and $j$ having two or more edges. When $x$ approaches $\bar{x} \in \Sigma_{i j}$, then $x_{i}>0$ and $x_{j}<0$, hence $\left|x_{j}\right|-x_{i}+\left|x_{i}\right|+x_{j}=0$, which means that $\nabla \sigma_{i j}(\bar{x}) \cdot h(\bar{x})>0$, i.e., in the case $\operatorname{sgn}\left(x_{i} x_{j}\right)=$ -1 the discontinuity surface is always crossed when it is approached from $\Sigma_{i j}^{-}$. An analogous argument holds when $\Sigma_{i j}$ is approached from $\Sigma_{i j}^{+}$. Hence as long as transitions are "simple" (i.e., in $\bar{x}$ only one of the $\Sigma_{i j}$ is crossed) and the crossing does not result in both nodes $i$ and $j$ becoming completely disconnected, the solution exists and it is unique.

When instead both nodes $i$ and $j$ do not have any other connection in $\Gamma_{s}(x)$ than the edge $(i, j)$, then since at the transition the edge disappears it becomes $h_{i}(\bar{x})=0$ and $h_{j}(\bar{x})=0$, i.e., $\nabla \sigma_{i j}(\bar{x}) \cdot h(\bar{x})=0$. This means that the solution stays on the surface $\Sigma_{i j}$ thereafter. Also in this case, however, the solution exists and it is unique. Notice that it is enough that one of the two nodes $i$ and $j$ has at least another edge to guarantee that $\nabla \sigma_{i j}(\bar{x}) \cdot h(\bar{x})>0$ at the transition. $\mathrm{P} 2$. Follows directly from condition 3 of the definition of proper initial conditions.

P3. Follows from Remark 1.

P4. A counterexample is in Example 1.
P5. A counterexample is in Example 3 below.

P6. A counterexample is in Example 4 below.

P7. As we are interested only in solutions issuing from a proper initial condition, it is enough to consider the case $\mathcal{I}_{0}=\emptyset$. The proof is similar for what is possible to that of Theorem 2 of [5]. At $t$ let us assume the components of $x(t)$ obey the following: $0<\left|x_{1}(t)\right| \leqslant \ldots \leqslant\left|x_{n}(t)\right|$ (notice that the order of absolute values can change over time). From (10) one has:

$$
\frac{d}{d t}\left|x_{i}\right|=\sum_{\substack{j \text { s.t. } \\\left|x_{i}-x_{j}\right|<1}}\left(\left|x_{j}\right|-\left|x_{i}\right|\right)
$$

When the expression (16) is computed for $x_{n}$ then $\frac{d}{d t}\left|x_{n}\right| \leqslant$ 0 , hence all $\left|x_{i}(t)\right|$ are bounded for all $t \geqslant 0$. Let us observe that when $\mathcal{I}_{0}=\emptyset$, because of symmetry, the following partial sums vanishes:

$$
\sum_{i=1}^{k} \sum_{\substack{j \leqslant k \text { s.t. } \\\left|x_{i}-x_{j}\right|<1}}\left(\left|x_{j}\right|-\left|x_{i}\right|\right)=0 .
$$

Hence, almost always

$$
\frac{d}{d t}\left|x_{i}\right|=\sum_{i=1}^{k} \sum_{\substack{j>k \text { s.t. } \\\left|x_{i}-x_{j}\right|<1}}\left(\left|x_{j}\right|-\left|x_{i}\right|\right) \geqslant 0
$$

because $j>k \geqslant i$ implies $\left|x_{j}\right| \geqslant\left|x_{k}\right| \geqslant\left|x_{i}\right|$. From the boundedness of $\left|x_{i}\right|$ (and of $\sum_{i=1}^{k}\left|x_{i}\right|$ ), it follows that $\left|x_{i}\right|$ must converge monotonically, and hence so must the $x_{i}$ almost always. To show that in $x^{*}$ either $x_{i}^{*}=x_{j}^{*}$ or $\left|x_{i}^{*}-x_{j}^{*}\right| \geqslant 1$, the same contradictory argument of [5] can now be used.

P8. From (16) and (17), if $\mathcal{I}_{0}=\emptyset$ then $\sum_{i=1}^{n} \frac{d}{d t}\left|x_{i}\right|=0$, hence $c_{s}(t)=$ const $\forall t \geqslant 0$.

P9. From Proposition 4 , if $x_{i}(0)=0$, then the following equivalent of (14) locally holds:

$$
\dot{x}_{i}=\left\{\begin{array}{l}
h_{i}^{+}(x)=\sum_{\substack{j \neq i \text { s.t. } \\
\left|x_{i}-x_{j}\right|<1}}\left|x_{j}\right| \\
h_{i}^{0}(x)=0 \\
h_{i}^{-}(x)=-\sum_{\substack{j \neq i \text { s.t. } \\
\left|x_{i}-x_{j}\right|<1}}\left|x_{j}\right| .
\end{array}\right.
$$

The solution corresponding to (18b) exists locally, from Proposition 4, and, by the same argument used in P1, can be extended to a Carathéodory solution on the entire half-line. The result follows trivially from (18b).

Remark 3 Although it obeys P8, the model (15) does not obey the equivalent of P4, i.e., $\frac{d W_{s}}{d t}$ need not be negative almost always. See Example 1 (blue dashed curve in Fig. 1).

Remark 4 Unlike in the unbounded case (Proposition 4), in P9 the solution corresponding to (18b) is only locally convergent to the origin. Furthermore it need not be a classical solution. 
Example 3 Consider the model (15) with the $n=3$ initial opinions $x(0)=\left[\begin{array}{lll}0.1 & -0.85 & -0.89\end{array}\right]^{T}$ and evolution shown in Fig 2 left panel. At $t=0, \Gamma(A(x))$ is fully connected, however $x_{1}$ (blue) becomes disconnected from $x_{3}$ already at $t=0.01$, and also from $x_{2}$ at $t=0.35$, i.e., P5 does not hold.
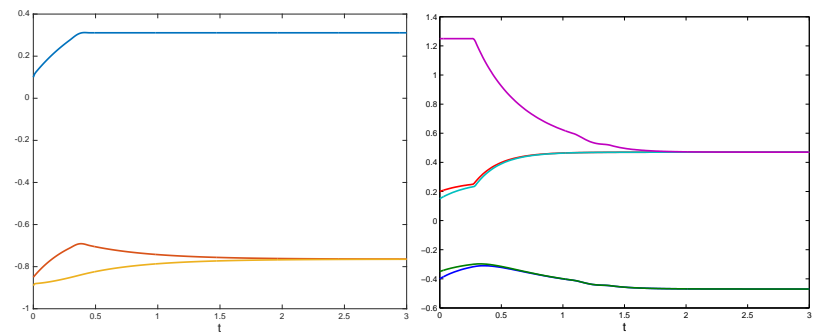

Fig. 2. Left: Example 3. The graph $\Gamma$ is fully connected at $t=0$ but $x_{1}$ becomes disconnected at later times. Right: Example 4. For the model (15) property P6 does not hold. In fact, one of the $n=5$ agents is disconnected from the remaining 4 at $t=0$, but all become connected at later $t$.

Example 4 Consider the model (15) with $n=5$ initial opinions $x(0)=\left[\begin{array}{lllll}-0.4 & -0.35 & 0.2 & 0.15 & 1.25\end{array}\right]^{T}$. For it, the property P6 does not hold, i.e., a disconnected $\Gamma(A(x))$ can become connected because of the repulsion, see Fig. 2, right panel.

Example 5 In Fig. 3, various possible outcomes of the clustering obtained with the model (15) are shown. If the $n=100$ agents have all nonzero initial conditions, then they will converge to bipartite bounded consensus, keeping the same sign (top left panel). None of them will approach 0. If one of the initial conditions is equal to 0 , then the nearest positive and negative groups of agents can converge to the origin (top right panel). However, it can also happen that only a group on one side converges to 0 (bottom left panel) or that no group at all does (bottom right panel). Fig. 3 shows that for the model (15) there is a sufficiently neat separation of time scales between the consensus-like convergence within a group and the convergence of a group to the origin due to diagonal dominance, when it happens.

\section{CONCLUSION}

The signed bounded confidence model proposed in this paper combines the clustering behavior of a standard bounded confidence model with sign invariance of the agents' opinions. Alternative models achieving the same goal exist and will be investigated in a future paper.

From a dynamical system viewpoint, it is worth pointing out the convergent behavior of one of the solutions corresponding to one or more of the initial opinions placed in the origin. Given that the signed graphs are by construction structurally balanced, this is an intrinsically nonlinear phenomenon, due to the presence of discontinuities induced by the sign functions, and with no counterpart in linear bipartite consensus (and in fact the solution remaining on
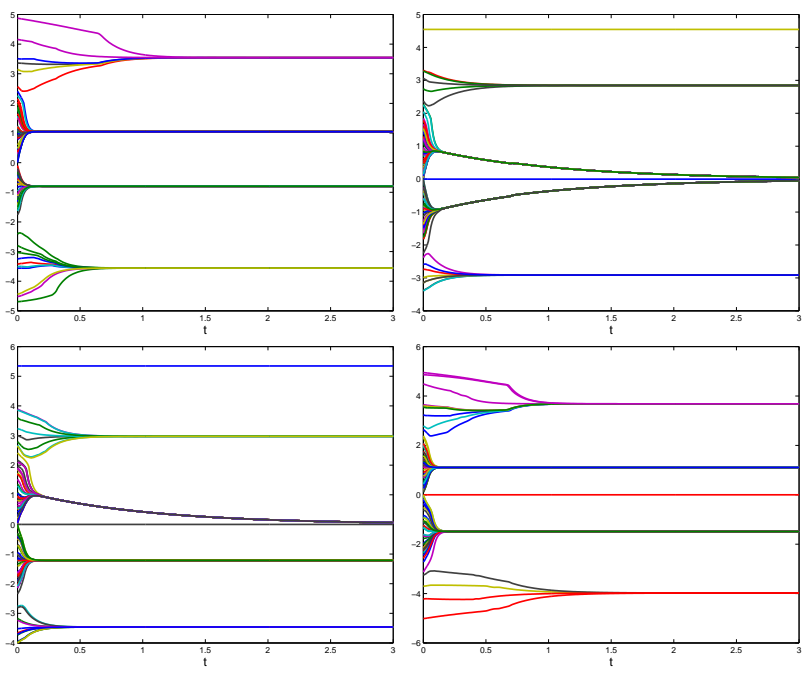

Fig. 3. Example 5.

the discontinuity surface is only one of the multiple solutions existing in this case).

\section{REFERENCES}

[1] C. Altafini. Consensus problems on networks with antagonistic interactions. IEEE Transactions on Automatic Control, 58(4):935-946, 2013.

[2] C. Altafini. Stability analysis of diagonally equipotent matrices. Automatica, 49(9):2780-2785, 2013.

[3] V.D. Blondel, J.M. Hendrickx, and J.N. Tsitsiklis. Existence and uniqueness of solutions for a continuous-time opinion dynamics model with state-dependent connectivity. Technical report, http://web.mit.edu/jnt/www/Papers/BHT10-solutions-DA.pdf, 2009.

[4] V.D. Blondel, J.M. Hendrickx, and J.N. Tsitsiklis. On Krause's multiagent consensus model with state-dependent connectivity. Automatic Control, IEEE Transactions on, 54(11):2586 -2597, nov. 2009.

[5] V.D. Blondel, J.M. Hendrickx, and J.N. Tsitsiklis. Continuous-time average-preserving opinion dynamics with opinion-dependent communications. SIAM Journal on Control and Optimization, 48(8):52145240, 2010.

[6] Francesca Ceragioli and Paolo Frasca. Continuous and discontinuous opinion dynamics with bounded confidence. Nonlinear Analysis: Real World Applications, 13(3):1239 - 1251, 2012.

[7] G. Como and F. Fagnani. Scaling limits for continuous opinion dynamics systems. Annals of Applied Probability, 21(4):1537-1567, 2011.

[8] J. Cortes. Discontinuous dynamical systems. Control Systems, IEEE, 28(3):36-73, June 2008.

[9] P. Frasca, H. Ishii, C. Ravazzi, and R. Tempo. Distributed randomized algorithms for opinion formation, centrality computation and power systems estimation: A tutorial overview. European Journal of Control, 24:2-13, 2015.

[10] Paolo Frasca, Chiara Ravazzi, Roberto Tempo, and Hideaki Ishii. Gossips and prejudices: ergodic randomized dynamics in social networks. In 4th IFAC Workshop on Distributed Estimation and Control in Networked Systems, volume 4, pages 212-219. International federation of Automatic Control, September 2013.

[11] N.E. Friedkin. The problem of social control and coordination of complex systems in sociology: A look at the community cleavage problem. IEEE Control Systems, 35(3):40-51, 2015.

[12] Rainer Hegselmann and Ulrich Krause. Opinion dynamics and bounded confidence models, analysis and simulation. Journal of Artificial Societies and Social Simulation, 5(3), 2002.

[13] Y. Yang, D.V. Dimarogonas, and X. Hu. Opinion consensus of modified Hegselmann-Krause models. Automatica, 50(2):622-627, 2014. 